\title{
Rechtspolitische Rahmenbedingungen für Prosumer
}

\begin{abstract}
Das Energierecht ist mittlerweile zu einem derart komplexen Werk an Gesetzen und Verordnungen angewachsen, dass es schwierig ist, alle Vorschriften herauszufiltern, die Prosumer besonders betreffen. Der Beitrag gibt einen groben Überblick über die aus Sicht der Autoren wichtigen Regelungen. Von Simon Schäfer-Stradowsky und Sandra Bachmann
\end{abstract}

Z B Beginn ist auf die Eigenheit einzugehen, dass es trotz der vielen Vorschriften im Energierecht bisher keine gesetzliche Definition für das Phänomen der Prosumer gibt. Gemein ist allen betreffenden Vorschriften, dass sie Privilegien an Prosumer adressieren, insbesondere Befreiungen von Abgabeund Umlagelasten beim Stromverbrauch.

Speziell die Erzeugung und der Verbrauch von Strom aus Erneuerbaren Energien (EE) vor Ort konnten bisher von vielen dieser Privilegien profitieren, die die dezentrale Energiewende gezielt anreizen sollen. Dies gilt vor allem für die Netzentgelte und die netzentgeltbezogenen Strompreisbestandteile (wie Kraft-Wärme-Kopplung-, Offshore- und StromNEV-Umlage, Konzessionsabgabe), für die die Zahlungspflicht bisher grundsätzlich entfällt, sofern der Strom nicht über das Netz der allgemeinen Versorgung zur Verbrauchsstelle geleitet wird.

\section{Status quo}

Spätestens mit der Reform des Erneuerbare-Energien-Gesetzes (EEG) im Jahr 2014 wurde allerdings ein klarer Paradigmenwechsel eingeläutet. Waren viele Prosumer bis 2014 von der EEG-Umlage noch vollständig befreit, so sieht das EEG nunmehr auch für sie grundsätzlich die volle Umlagepflicht vor. Eine Ausnahme von diesem Grundsatz für Neuanlagen gibt es nur noch in Form der anteiligen Befreiung von der EEG-Umlage unter den engen Voraussetzungen der Eigenversorgung nach Paragraf (『) 5 Nr.12 EEG für Strom aus EE-Anlagen oder hocheffizienten Kraft-Wärme-Kopplung-Anlagen, siehe \61 Abs. 1 EEG. Weiterhin vollständig von der EEG-Umlage befreit sind gemäß $\int 61$ Abs. 2 EEG-Kleinanlagen bis zehn Megawattstunden pro Jahr, der Kraftwerkseigenverbrauch, Inselanlagen und die vollständige Eigenversorgung mit Strom aus EE ohne Inanspruchnahme einer Förderung für Überschussstrom. Um in den Genuss dieser Privilegien zu kommen, muss der Pro- sumer allerdings Kosten auf sich nehmen, denn er muss seinen Darlegungs- und Mitteilungspflichten nach Paragrafen (IV) 60, 61, 74 EEG und $\iint 7$ und 9 der Ausgleichsmechanismusverordnung gegenüber den Netzbetreibern nachkommen. Dementsprechend hat er seine Erzeugungs- und Verbrauchswerte durch geeichte Messeinrichtungen viertelstundenscharf zu ermitteln, siehe $\int 61$ Abs. 6 und 7 EEG.

Probleme entstehen vor allem dann, wenn der Prosumer mehr Strom erzeugt, als er selber verbraucht, und diesen in das Netz einspeisen möchte. Will der Prosumer seinen Überschussstrom in einem mittelbar oder unmittelbar an das Netz angeschlossenen Stromspeicher zwischenspeichern, so wird dieser Vorgang für die meisten Speichertechnologien nach dem Wortlaut nicht von dem Befreiungstatbestand des \60 Abs. 3 S. 1 EEG erfasst. Da die Einspeicherung derzeit auch bei der Eigenversorgung als Letztverbrauch angesehen wird, ist für diesen Vorgang von typischen Prosumern die volle EEG-Umlage zu zahlen. Hindernisse stellen in diesem Zusammenhang auch die Verpflichtung zur Direktvermarktung für Neuanlagen ab 100 Kilowatt ( $\ 37$ Abs. 2 Nr. 2 EEG) und der Ausschluss von der Teilnahme am Regelenergiemarkt dar, die derzeit nur über den Umweg eines Dienstleisters möglich ist.

Neben diesen Vorgaben des EEG kommt eine Privilegierung von Prosumern auch im Rahmen einer Stromsteuerbefreiung in Betracht. Eine Befreiung ist für den Vor-Ort-Stromverbrauch aus EE-Anlagen bis zwei Megawatt nach $\int 9$ Abs. 1 Nr. 3 a StromStG möglich. Zudem ist nach \9 Abs. 1 Nr. 1 StromStG Strom von der Stromsteuer befreit, der ausschließlich aus mit erneuerbaren Energieträgern gespeisten Netzen entnommen wird („grüner Strom aus grünen Netzen“). Allerdings sind diese Regeln sehr restriktiv, denn die Privilegierung ist auf eine bestimmte Anlagengröße beschränkt beziehungsweise setzt EE-Netze voraus.

\section{Aktuelle Entwicklungen}

Von den vielen Gesetzesvorhaben, die im Energierecht in letzter Zeit angestoßen wurden, ist das Kraft-Wärme-Kopplungsgesetz (KWKG) schon am weitesten vorangeschritten seit dem 1.1.2016 gilt das KWKG 2016. Dieses birgt teilweise Verschlechterungen für Prosumer, da nun auch im KWKG 2016 die Direktvermarktung als verpflichtend eingeführt wurde, was für die Vermarktung von Überschussstrom schwierig sein könnte. Zudem gilt die Förderung der Eigenversorgung gemäß 『6 Abs. 4 S. 1 Nr. 1 KW KG 2016 nur noch für Kleinanlagen (bis 100 Kilowatt). 
Schließlich können sich für Prosumer weitreichende Einschränkungen aus der anstehenden EEG-Novelle 2016 ergeben, durch die insbesondere das Ausschreibungssystem für die meisten EE-Anlagen als Regelfall eingeführt werden soll. Nach dem derzeitigen Referentenentwurf des Bundesministeriums für Wirtschaft und Energie vom 29.02. 2016 ist vorgesehen, dass alle neuen Anlagen mit einer Leistung ab einem Megawatt zwingend an der Ausschreibung teilnehmen müssen. Gleichzeitig wird durch den geplanten neuen \27a EEG 2016 die Eigenversorgung im Rahmen der Ausschreibung ausgeschlossen.

Durch den geplanten 『61a Abs. 1 EEG 2016 verbessern sich hingegen die Bedingungen bei der Einspeicherung in bestimmte Stromspeicher. Die EEG-Umlage soll bei der Einspeicherung dann entfallen, wenn später für den verbrauchten Strom die EEG-Umlage gezahlt wird.

Ferner soll das EEG um die Vorschrift des \19 Absatz 1a EEG 2016 erweitert werden, die eine Kumulation von EEG-Förderung und Stromsteuerbefreiung rückwirkend zum 01.01.2016 verhindern soll. Der typische Prosumer müsste sich dann entscheiden, welche Förderung er in Anspruch nehmen möchte, sicherlich eine Verschlechterung für viele Prosumer. Noch ist unklar, ob es bei dieser geplanten Rückwirkung bleibt, da sie einen Verstoß gegen den Vertrauensschutz darstellen könnte.

Um den gleichzeitig steigenden Anforderungen bei den einzusetzenden Mess- und Kommunikationstechnologien und Datenverarbeitungssystemen gerecht zu werden, ist derzeit zudem das Digitalisierungsgesetz für intelligente Messsysteme (Smart-Meter-Rollout) in Planung. Ziel ist es vor allem, die volatil auftretenden Einspeisungen beherrschbar zu machen. Mit der teilweise vorgesehenen Einbaupflicht von Smart Metern für Erzeuger und Verbraucher erhalten die Prosumer im Gegenzug einen Informationsanspruch gegenüber den Messstellenbetreibern. Mithilfe dieser Informationen können Prosumer unter Umständen ihren Selbstverbrauch effizienter ausgestalten. Problematisch könnten aber die vorgesehenen Entflechtungsvorgaben gegenüber dem Messstellenbetreiber sein.

Neben dem Digitalisierungsgesetz liegt zudem ein Entwurf des Gesetzes zur Weiterentwicklung des Strommarktes (Strommarktgesetz) vor. Interessant sind hier vor allem der geplante Wegfall der vermiedenen Netzentgelte für Betreiber dezentraler Anlagen ( $\mathbb{1} 18$ Abs. 1 S. 1 StromNEV), wodurch bestimmte Prosumer bisher gefördert wurden, und der Abbau der Eintrittsbarrieren für EE-Anlagen im Regelleistungsmarkt über eine Änderung des $₫ 26$ Abs. 3 StromNZV, wodurch Prosumer verbesserte Bedingungen bei der Vermarktung ihres Überschussstroms erhalten könnten.

Nach alledem ist deutlich erkennbar, dass sich die Rahmenbedingungen für Prosumer zukünftig stark verändern werden. Insbesondere die geplanten Änderungen im EEG werden dazu führen, dass es immer weniger Prosumer im Bereich großer Erzeugungsanlagen geben wird. Nur noch Prosumer mit kleinen Anlagen, also vor allem Privathaushalte, sollen zukünftig privilegiert werden. Die bisher klar dezentrale Ausrichtung der Energiewende wird damit zunehmend infrage gestellt.

AUTOREN + KONTAKT

Simon Schäfer-Stradowsky ist Geschäftsführer des Instituts für Klimaschutz, Energie und Mobilität e. V. (IKEM)

IKEM, Magazinstraße 15-16, 10179 Berlin Tel.: +49 $30408187-010$

E-Mail: simon.schaefer-stradowsky@ikem.de

Sandra Bachmann war Rechtsreferendarin am IKEM
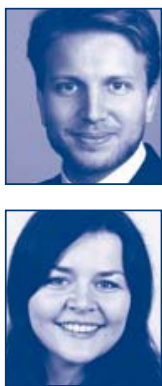

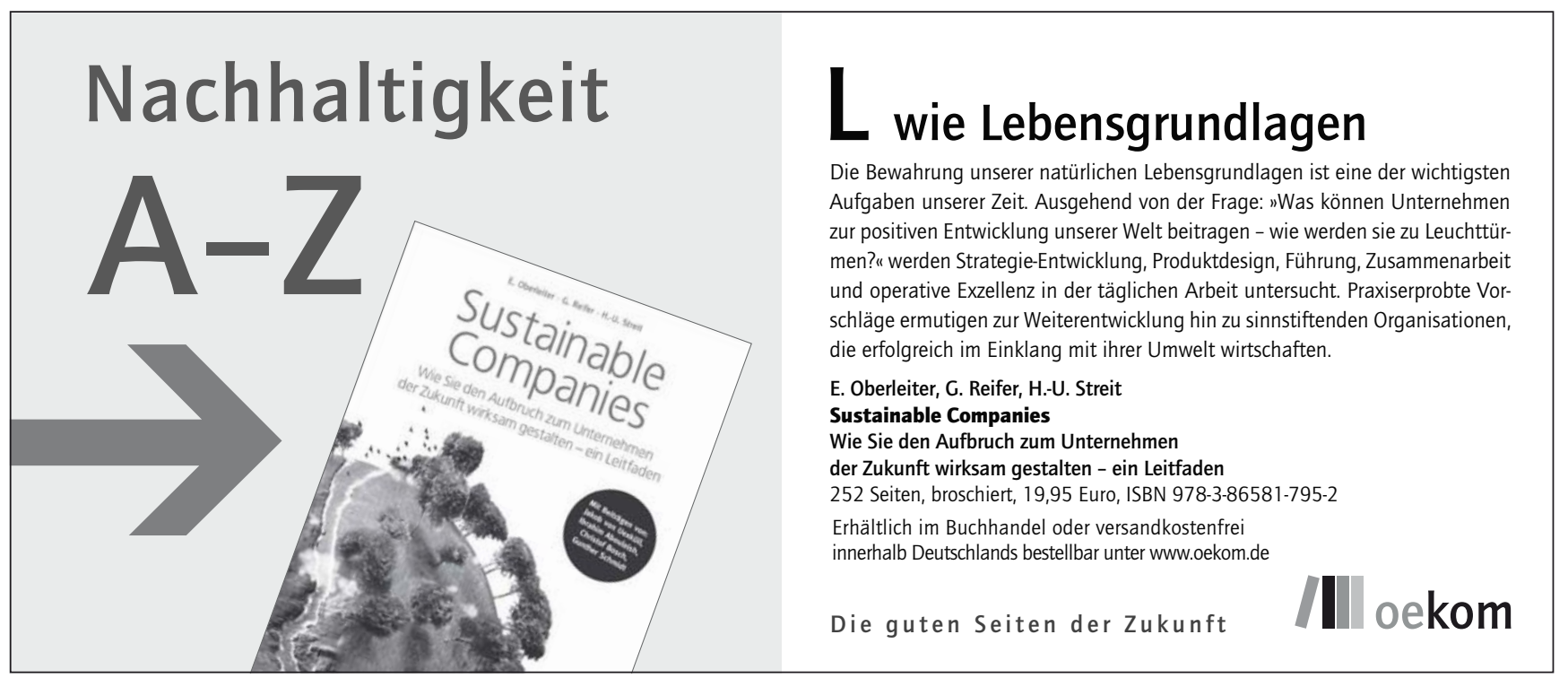

